\title{
ON THE TEMPERATURE STRUCTURE OF THE GALACTIC CENTER CLOUD G0.253+0.016
}

\author{
Paul C. Clark ${ }^{1}$, Simon C. O. Glover ${ }^{1}$, Sarah E. Ragan ${ }^{2}$, Rahul Shetty ${ }^{1}$, and Ralf S. Klessen ${ }^{1}$ \\ ${ }^{1}$ Zentrum für Astronomie, Institut für Theoretische Astrophysik, Universität Heidelberg, Albert-Ueberle-Str. 2, D-69120 Heidelberg, Germany; \\ p.clark@uni-heidelberg.de, glover@uni-heidelberg.de, r.shetty@uni-heidelberg.de, klessen@uni-heidelberg.de \\ ${ }^{2}$ Max Planck Institut für Astronomie, Königstuhl 17, D-69117 Heidelberg, Germany; ragan@ mpia.de \\ Received 2013 March 8; accepted 2013 April 3; published 2013 April 26
}

\begin{abstract}
We present a series of smoothed particle hydrodynamical models of G0.253+0.016 (also known as “The Brick"), a very dense molecular cloud that lies close to the Galactic center. We explore how its gas and dust temperatures react as we vary the strength of both the interstellar radiation field (ISRF) and the cosmic-ray ionization rate (CRIR). The cloud has an extent in the plane of the sky of roughly $3.4 \mathrm{pc} \times 9.4 \mathrm{pc}$. As its size along the line of sight is unknown, we consider two cases. In our fiducial, high-density model, we adopt a depth along the line of sight of $3.4 \mathrm{pc}$, and in the low-density model we assume an extent along the line of sight of $17 \mathrm{pc}$. To recover the observed gas and dust temperatures, we find that the ISRF must be around 1000 times the solar neighborhood value, and the CRIR must be roughly $10^{-14} \mathrm{~s}^{-1}$, regardless of the geometries studied. For such high values of the CRIR, we find that cooling in the cloud's interior is dominated by neutral oxygen, in contrast to standard molecular clouds, which at the same densities are mainly cooled via CO. Our results suggest that the conditions near G0.253+0.016 are more extreme than those generally accepted for the inner 500 pc of the galaxy.
\end{abstract}

Key words: cosmic rays - evolution - Galaxy: center - ISM: molecules

Online-only material: color figures

\section{INTRODUCTION}

The environmental conditions in the Galactic center (GC) provide an extreme test of our current understanding of the star formation process (e.g., Papadopoulos 2010; Krumholz et al. 2012; Longmore et al. 2013; Kruijssen et al. 2013). With both stronger background radiation fields and higher cosmic-ray (CR) fluxes compared to clouds in the solar neighborhood, star formation is predicted to occur at higher volume and column densities than is typical in a standard giant molecular cloud (Elmegreen et al. 2008).

One notable example is G0.253+0.016 (also referred to as the "The Brick"), which displays both extremely high column and volume densities, yet very little sign of star formation (Güesten et al. 1981; Lis et al. 1994; Longmore et al. 2012). Despite the current lack of star formation, the physical conditions in this object are thought to be similar to those required for the formation of massive stellar clusters (Longmore et al. 2012).

In this Letter, we investigate the influence of the extreme GC environment on the thermodynamics of dense and massive molecular clouds, in an attempt to better understand the initial conditions for star formation in the inner molecular zone. We adopt values for the interstellar radiation field (ISRF) and the cosmic-ray ionization rate (CRIR) that are significantly higher than those measured in solar neighborhood molecular clouds. For more fundamental parameters such as the mass, dimensions, and turbulent velocity dispersion of the clouds, we take the values for $\mathrm{G} 0.253+0.016$ reported by Longmore et al. (2012). In contrast to the other clouds in the GC, the apparent lack of star formation in G0.253+0.016 makes it an ideal candidate for studying the effects of the environmental conditions on the thermal balance of the cloud.

\section{COMPUTATIONAL METHOD}

We perform our simulations using the smoothed particle hydrodynamics (SPH) code GADGET2 (Springel 2005). We have modified the code to include time-dependent chemistry and a treatment of the main heating and cooling processes (described below). We have also included an implementation of the TREECoL algorithm (Clark et al. 2012) to obtain column density maps of the sky as seen by each SPH particle. These maps (including total, $\mathrm{H}_{2}$ and $\mathrm{CO}$ column densities) are used to calculate the influence of the ISRF on the gas and the dust.

We assume for simplicity that the spectral shape of the ISRF follows Draine (1978) in the UV and Black (1994) at longer wavelengths. We denote the solar neighborhood value of the strength of the ISRF as $G_{0}$, and perform simulations with field strengths $100 G_{0}$ and $1000 G_{0}$ (see Table 1). Note that this multiplicative scaling is done equally at all wavelengths. For our dust model, we use a combination of values from Ossenkopf \& Henning (1994; non-coagulated, thick ice mantle grains) for wavelengths longer than $1 \mu \mathrm{m}$, and from Mathis et al. (1983) at shorter wavelengths. To compute the visual extinction, we use the relationship $A_{\mathrm{V}}=5.348 \times 10^{-22}\left(N_{\mathrm{H}, \text { tot }} / 1 \mathrm{~cm}^{-2}\right)$, where $N_{\mathrm{H} \text {,tot }}$ is the total hydrogen column density (Bohlin et al. 1978; Draine \& Bertoldi 1996). For simplicity, we do not account for any changes in the extinction curve that may occur due to dust coagulation. For the CRIR, we adopt a value of $I_{\mathrm{CR}, 0}=3 \times 10^{-17} \mathrm{~s}^{-1}$ as our solar neighborhood value (van der Tak \& van Dishoeck 2000), and assume that each ionization event deposits $20 \mathrm{eV}$ of energy into the gas (Goldsmith \& Langer 1978). The dependence of the CRIR on column density is highly uncertain (Padovani et al. 2009), and we assume for simplicity that no attenuation occurs. We do not include the effects of ionization by hard X-rays, as this does not appear to be a major heat source in the GC, given the relatively low X-ray luminosity (Rodríguez-Fernández et al. 2004; Schleicher et al. 2010)

For the chemistry we adopt the reduced CO network of Nelson \& Langer (1999). Details can be found in Glover \& Clark (2012b), and a description of how the chemistry interacts with the ISRF via the TREECOL algorithm is given in Glover \& Clark (2012a).

\section{INITIAL CONDITIONS AND MODEL PARAMETERS}

For the initial conditions in this study, we take the cloud properties derived in Longmore et al. (2012) for G0.253+0.016 
Table 1

Summary of the Simulations

\begin{tabular}{|c|c|c|c|c|c|c|c|c|c|c|c|}
\hline Model & $\begin{array}{c}L_{x} \\
(\mathrm{pc})\end{array}$ & $\begin{array}{c}L_{y} \\
(\mathrm{pc})\end{array}$ & $\begin{array}{c}L_{z} \\
(\mathrm{pc})\end{array}$ & $\begin{array}{c}\Sigma_{\min , 0} \\
\left(\mathrm{~cm}^{-2}\right)\end{array}$ & $\begin{array}{c}n_{0} \\
\left(\mathrm{~cm}^{-3}\right)\end{array}$ & $\begin{array}{l}I_{\mathrm{ISRF}} \\
\left(G_{0}\right)\end{array}$ & $\begin{array}{c}I_{\mathrm{CR}} \\
\left(\mathrm{s}^{-1}\right)\end{array}$ & $x\left(\mathrm{H}_{2}\right)$ & $x(\mathrm{CO})$ & $x\left(\mathrm{C}^{+}\right)$ & $x(\mathrm{O})$ \\
\hline 1 & 9.4 & 3.4 & 3.4 & $3.6 \times 10^{23}$ & $3.5 \times 10^{4}$ & 1000 & $3 \times 10^{-14}$ & 0.477 & $1.5 \times 10^{-5}$ & $1.33 \times 10^{-5}$ & $3.06 \times 10^{-4}$ \\
\hline 2 & 9.4 & 3.4 & 3.4 & $3.6 \times 10^{23}$ & $3.5 \times 10^{4}$ & 100 & $3 \times 10^{-15}$ & 0.500 & $9.59 \times 10^{-5}$ & $8.02 \times 10^{-6}$ & $2.24 \times 10^{-4}$ \\
\hline 3 & 9.4 & 3.4 & 3.4 & $3.6 \times 10^{23}$ & $3.5 \times 10^{4}$ & 1000 & $3 \times 10^{-16}$ & 0.500 & $1.11 \times 10^{-4}$ & $3.08 \times 10^{-6}$ & $2.01 \times 10^{-4}$ \\
\hline 4 & 9.4 & 3.4 & 17.0 & $7.3 \times 10^{22}$ & $6.7 \times 10^{3}$ & 100 & $3 \times 10^{-16}$ & 0.500 & $6.65 \times 10^{-5}$ & $1.76 \times 10^{-5}$ & $2.53 \times 10^{-4}$ \\
\hline 5 & 9.4 & 3.4 & 17.0 & $7.3 \times 10^{22}$ & $6.7 \times 10^{3}$ & 100 & $3 \times 10^{-15}$ & 0.496 & $2.62 \times 10^{-5}$ & $2.41 \times 10^{-5}$ & $2.94 \times 10^{-4}$ \\
\hline 6 & 9.4 & 3.4 & 17.0 & $7.3 \times 10^{22}$ & $6.7 \times 10^{3}$ & 1000 & $3 \times 10^{-16}$ & 0.497 & $6.10 \times 10^{-5}$ & $2.52 \times 10^{-5}$ & $2.59 \times 10^{-4}$ \\
\hline
\end{tabular}

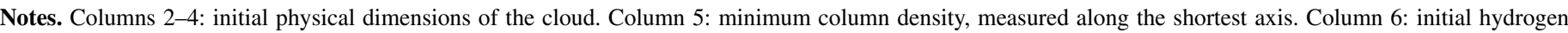

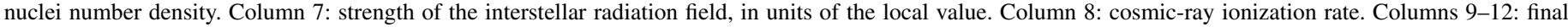

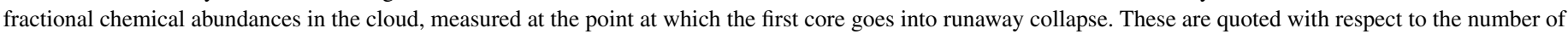
$\mathrm{H}$ nuclei. A fully molecular gas therefore has $x\left(\mathrm{H}_{2}\right)=0.5$. The total carbon and oxygen abundances in the models are $1.4 \times 10^{-4}$ and $3.2 \times 10^{-4}$, respectively.

as a guide: a size of $9.4 \mathrm{pc} \times 3.4 \mathrm{pc}$, and a mass of $1.3 \times 10^{5} \mathrm{M}_{\odot}$. The clouds are simulated using $2 \times 10^{7} \mathrm{SPH}$ particles, and so our mass resolution is $M_{\text {res }}=0.65 M_{\odot}$ (Hubber et al. 2006). We adopt a simple rectangular cuboid geometry, matching the longer of the two observed dimensions with the $x$-axis in the simulations, and the shorter with the $y$-axis, such that all the clouds have particles placed initially from 0 to $9.4 \mathrm{pc}$ in $x\left(L_{x}\right)$ and 0 to $3.4 \mathrm{pc}$ in $y\left(L_{y}\right)$. In the $z$-direction, we adopt two values for the extent of the cloud, since the true dimension of G0.253+0.016 along the line of sight is unknown. Our first choice is to make the $z$-axis the same length as the $y$-axis, yielding a mean hydrogen nuclei number density $n_{0}=3.5 \times 10^{4} \mathrm{~cm}^{-3}$. This is the setup used in our "fiducial" clouds. Our second choice is to make $z$ the longest axis, with $L_{z}=17.0 \mathrm{pc}$. These clouds have an initial density of $6.9 \times 10^{3} \mathrm{~cm}^{-3}$ and are our "low-density" clouds. All the clouds are given non-thermal support in the form of a turbulent velocity field, which has a power spectrum $P(k) \propto k^{-4}$. The turbulence is permitted to decay as the cloud evolves. We fix the initial three-dimensional (3D) turbulent velocity dispersion based on the observational data: Longmore et al. (2012) report a line width of $15.1 \mathrm{~km} \mathrm{~s}^{-1}$ for G0.253+0.016, equivalent to a onedimensional velocity dispersion of $6.4 \mathrm{~km} \mathrm{~s}^{-1}$, and hence to a $3 \mathrm{D}$ velocity dispersion of $11.12 \mathrm{~km} \mathrm{~s}^{-1}$, assuming isotropic turbulence.

We perform three simulations for each of our two cloud models, varying the strength of the ISRF and the magnitude of the CRIR. An overview of the simulations can be found in Table 1. A central assumption here is that the shape of the radiation field and the CR energy spectrum are the same locally and in the GC, and that it is only the normalization of each that changes.

In view of the high densities probed by our initial conditions, we assume that the hydrogen in our clouds starts as $\mathrm{H}_{2}$. However, we start with carbon in the form of $\mathrm{C}^{+}$, and allow it to selfconsistently evolve to form $\mathrm{C}$ and $\mathrm{CO}$. As we discuss in Section 5 , the clouds are already in chemical equilibrium at the point at which we perform our analysis.

\section{GAS AND DUST TEMPERATURE}

Using Herschel observations, Longmore et al. (2012) show that the dust temperature varies smoothly from $19 \mathrm{~K}$ in the cloud center to $27 \mathrm{~K}$ at the edge. Observational constraints on the gas temperature of G0.253+0.016 have existed for some time: Güesten et al. (1981) derive rotation temperatures of $\sim 45 \mathrm{~K}$ using ammonia transitions, corresponding to an average kinetic temperatures of $\sim 80 \mathrm{~K}$ (Walmsley \& Ungerechts 1983). A recent formaldehyde survey (Ao et al. 2013) finds average kinetic temperatures of $65-70 \mathrm{~K}$, which agrees within the uncertainties. However, observations of high-excitation ammonia lines suggest that G0.253+0.016 has a complex gas temperature structure, with components up to $400 \mathrm{~K}$, that has yet to be modeled (E. Mills, 2013, private communication). What environmental conditions are required to produce such temperatures?

The typical features of our cloud are illustrated in Figure 1, which shows the column densities of one of the clouds in the $x-y$ plane (i.e., integrating along $z$ ), and the accompanying mean gas and dust temperature maps. This cloud is our most extreme case studied, with $I_{\mathrm{ISRF}}=1000 \mathrm{G}_{0}, I_{\mathrm{CR}}=1000 I_{\mathrm{CR}, 0}$, and our "fiducial" cloud geometry. However, the features of this cloud are mirrored in our other simulations - the clouds have a hot skin and a relatively cool interior, and are highly structured by the supersonic turbulence. The images in Figure 1 are taken just as the first collapsing core exceeds a density of around $10^{8} \mathrm{~cm}^{-3}$, and so represent the state of the cloud at the onset of star formation. All the other clouds in this study will be presented at the same point in their evolution.

In Figure 2, we show the gas and dust temperatures in the clouds as a function of the position along the $x$-axis. The most obvious feature of these profiles is that the gas and dust have different temperatures throughout the cloud. They are not thermodynamically coupled on the scales shown here, consistent with the observations mentioned above.

The profiles also reveal how the environment affects the cloud temperature. We see that the CRs are responsible for heating the gas, while the ISRF is primarily responsible for heating the dust. Such a result is expected. The high column density of this cloud means that photoelectric emission in the cloud interior is strongly suppressed, as the UV photons responsible for it are readily absorbed near the surface of the cloud. As such, the ISRF can play only a minor role in directly heating the gas. On the other hand, as the CRs have no attenuation in our model, they are free to heat the cloud's gaseous interior throughout. The ISRF can, however, heat the dust at the center of the cloud, as this heating comes primarily from longer wavelength photons, which are able to penetrate much further than the UV photons. In summary, for clouds with such an extreme column density as $\mathrm{G} 0.253+0.016$, the heating of the dust and gas is effectively split into two components.

Our 3D modeling results suggest that for our fiducial cloud model, the environmental parameters that best reproduce the observed temperatures are $I_{\mathrm{ISRF}}=1000 G_{0}$ and 

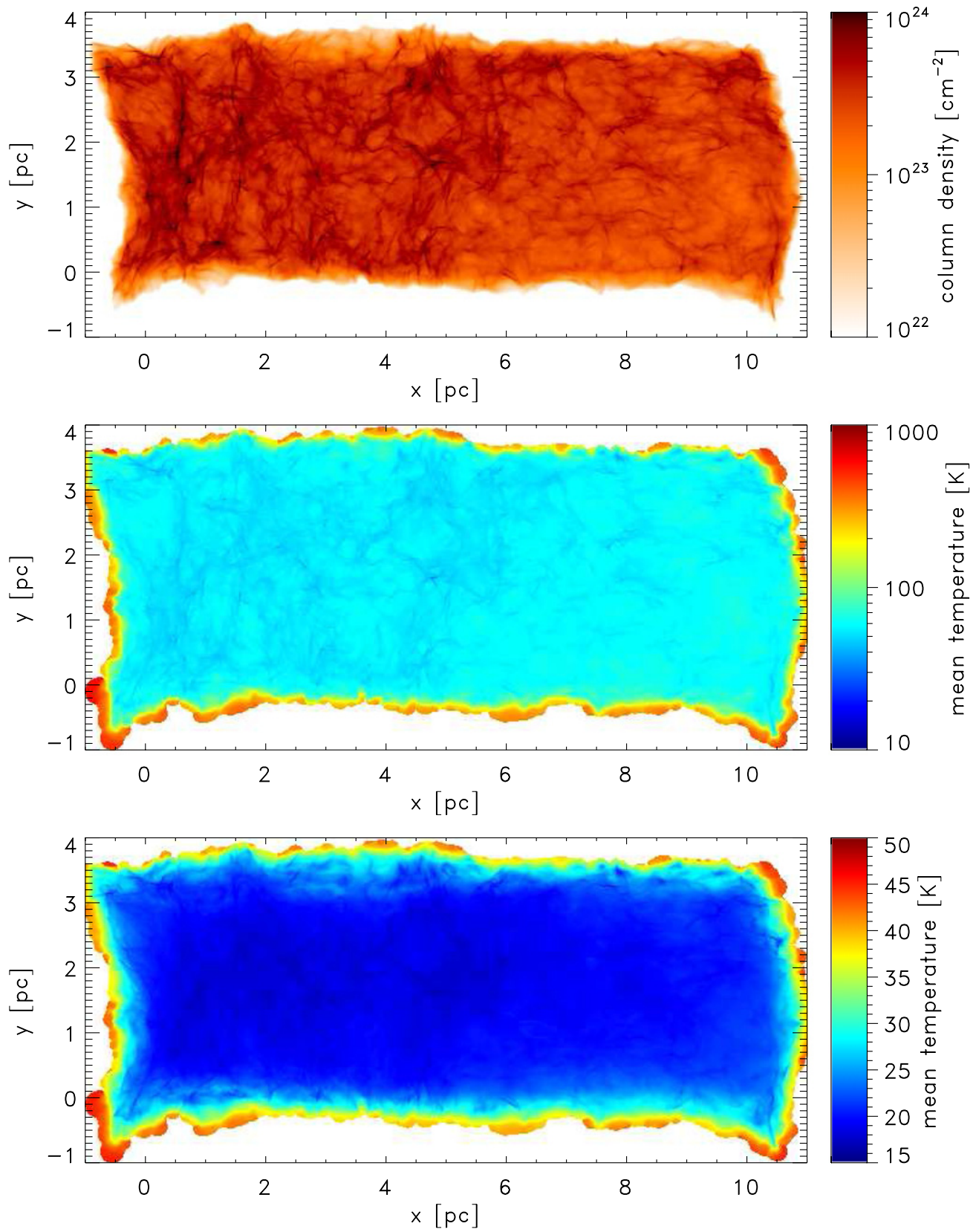

Figure 1. Column density, and mean gas and dust temperatures in our fiducial cloud setup (simulation "1" in Table 1), with the ISRF set at $1000 G_{0}$ and the CRIR at $3 \times 10^{-14} \mathrm{~s}^{-1}$.

(A color version of this figure is available in the online journal.)

$I_{\mathrm{CR}}=1000 I_{\mathrm{CR}, 0}$. Reducing either of these values by a factor of 10 results in gas or dust temperatures that are too low to agree with the observations.

One potential source of error is simply that we have underestimated the extent of G0.253+0.016 along the observed line of sight, and so the true effective column of the cloud is much smaller than we are assuming in the fiducial models. However, we find that similar environmental conditions are also required when we consider our lower-density version of G0.253+0.016. These models are shown on the bottom row of Figure 2. Even in these lower column density clouds, we see that the ISRF is mainly responsible for determining the dust temperatures (i.e., there is very little gas-dust thermodynamic coupling), and the CRIR is mainly responsible for determining the gas temperatures. Our dust temperatures are now a little higher than the observed values throughout the cloud, suggesting that for this geometry the $I_{\mathrm{ISRF}}$ would need to be lower than $1000 G_{0}$. However, we see that by $100 G_{0}$ the ISRF is already too low to explain the observed temperatures. Also, we see that $I_{\mathrm{CR}}=100$ $I_{\mathrm{CR}, 0}$ results in a gas temperature of around $30 \mathrm{~K}$ in the interior of the cloud-again, this is inconsistent with the observations.

Figure 2 also shows that the geometry of the cloud affects the temperature gradients along the cloud. This is particularly evident when one looks at the gas temperature, especially when $I_{\text {ISRF }}$ is high (see, e.g., the bottom right panel). This implies that it should be possible to constrain both the total ISRF and the cloud's geometry by fitting the gradient of the gas temperature in the cloud modeling. Such a study is worth revisiting, once maps of the gas temperature with sub-parsec resolution become available.

Finally, we note that both the gas and dust temperatures can vary considerably along a line of sight from the averages shown 

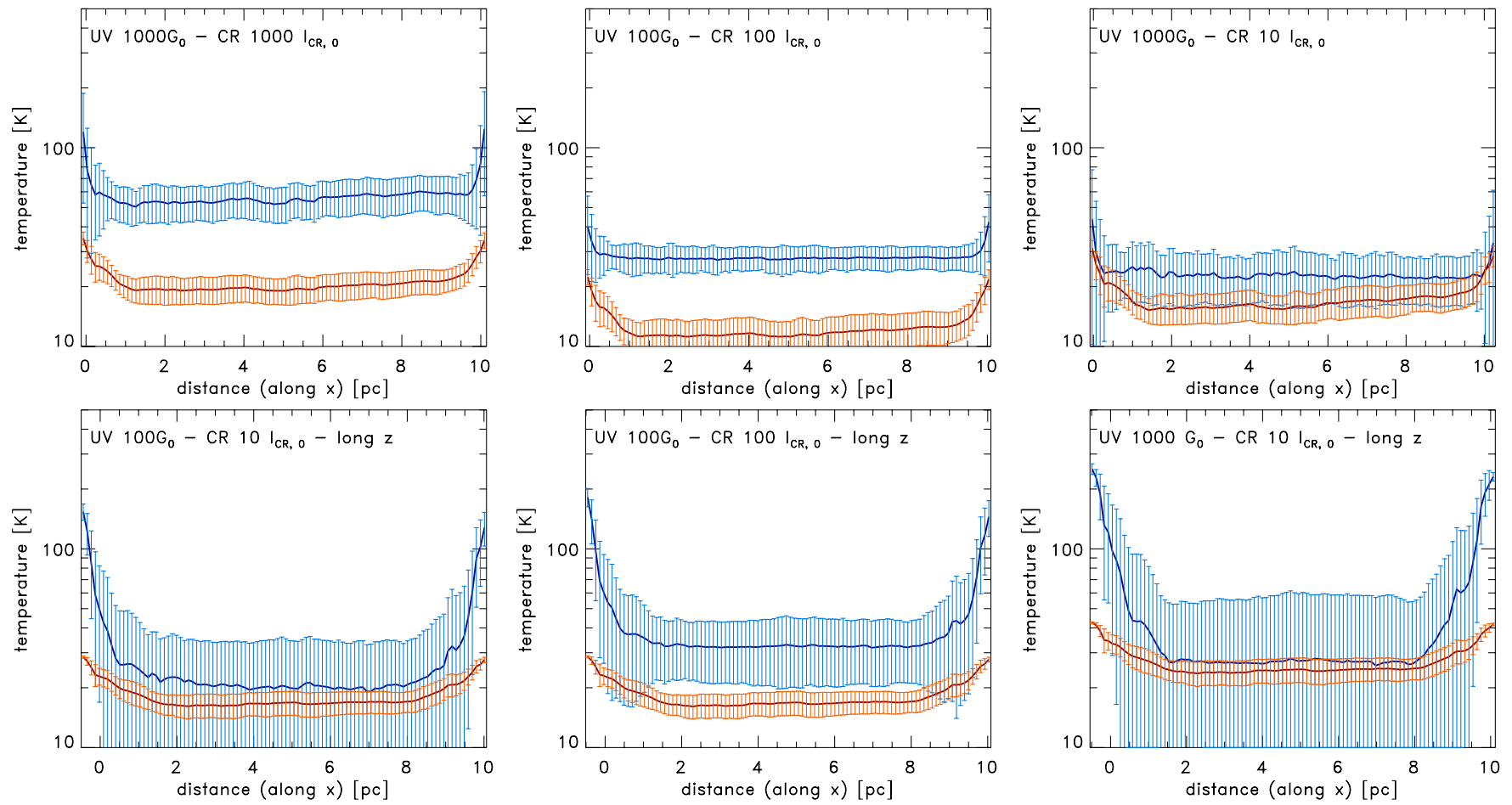

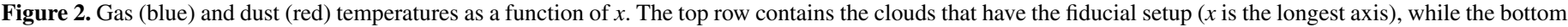

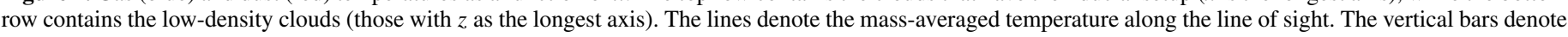
the $1 \sigma$ spread.

(A color version of this figure is available in the online journal.)

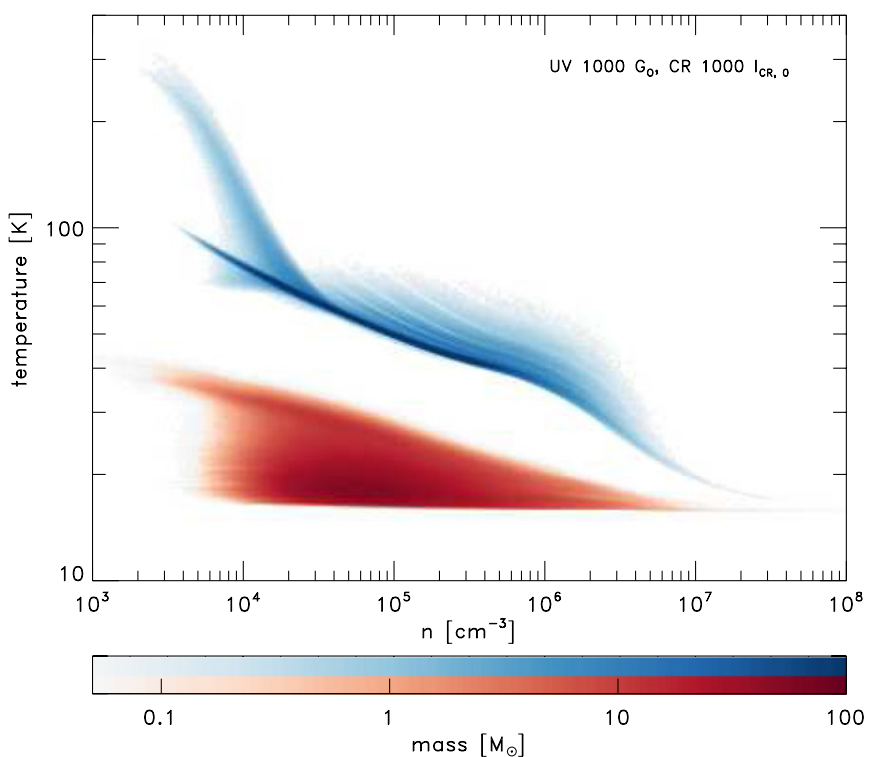

Figure 3. Gas (blue) and dust (red) temperatures as a function of density in our fiducial cloud (model " 1 " in Table 1).

(A color version of this figure is available in the online journal.)

in Figure 2. This can already be seen in the images in Figure 1. However, we also show in Figure 3 how the temperatures vary as a function of density in our fiducial case. We see that at high densities $\left(>10^{6} \mathrm{~cm}^{-3}\right)$, once the dust and gas thermally couple, the temperatures can be relatively cold.

\section{HEATING AND COOLING PROCESSES}

In this section we investigate the heating and cooling processes for the gas in more detail. The dominant processes that govern the gas temperature are shown as functions of density in Figure 4 for the two most extreme cases: our fiducial cloud $\left(n_{0}=\right.$ $3.5 \times 10^{4} \mathrm{~cm}^{-3}$ ), with $I_{\mathrm{ISRF}}=1000 \mathrm{G}_{0}$ and $I_{\mathrm{CR}}=1000 I_{\mathrm{CR}, 0}$, and one of the lower-density clouds $\left(n_{0}=6.7 \times 10^{3} \mathrm{~cm}^{-3}\right)$, with $I_{\mathrm{ISRF}}=100 \mathrm{G}_{0}$ and $I_{\mathrm{CR}}=10 I_{\mathrm{CR}, 0}$.

In both clouds, the dominant heating processes follow a broadly similar pattern. At the lowest densities, which represent the outskirts of the clouds in these simulations, the dominant heat source is photoelectric emission from dust grains. This falls off sharply as we move to higher densities as a result of the increasing extinction as one moves into the cloud's interior. At slightly higher densities, the heating caused by CRs starts to dominate the thermal balance. In the case of the hotter, denser cloud, this process remains the main heating source until we reach a number density $n=10^{8} \mathrm{~cm}^{-3}$, corresponding to our resolution limit. In the lower density cloud, embedded in the less extreme environment, shock heating becomes the main source of heat input to the gas at densities above $n \sim 10^{5} \mathrm{~cm}^{-3}$. Note that since neither compression nor shock heating is dominant in the high CRIR case, the temperature of the cloud cannot be used to determine its age.

When we compare the main cooling processes, we also find some similarities. In the low-density outskirts, where the gas is warm and there is little $\mathrm{CO}$, we find that $\mathrm{C}^{+}$and neutral oxygen emission are the main coolants, as in the low-density interstellar medium. Given the high densities and temperatures of the cloud's skin, and the fact that we start with the hydrogen in molecular form, we also find that $\mathrm{H}_{2}$ can be an effective coolant at the outskirts.

As we move into the cloud, however, the gas temperature drops and the $\mathrm{C}^{+}$recombines to form $\mathrm{C}$ and then $\mathrm{CO}$. The identity of the dominant coolant therefore changes. In the lowdensity cloud, $\mathrm{CO}$ cooling dominates in this slightly denser 

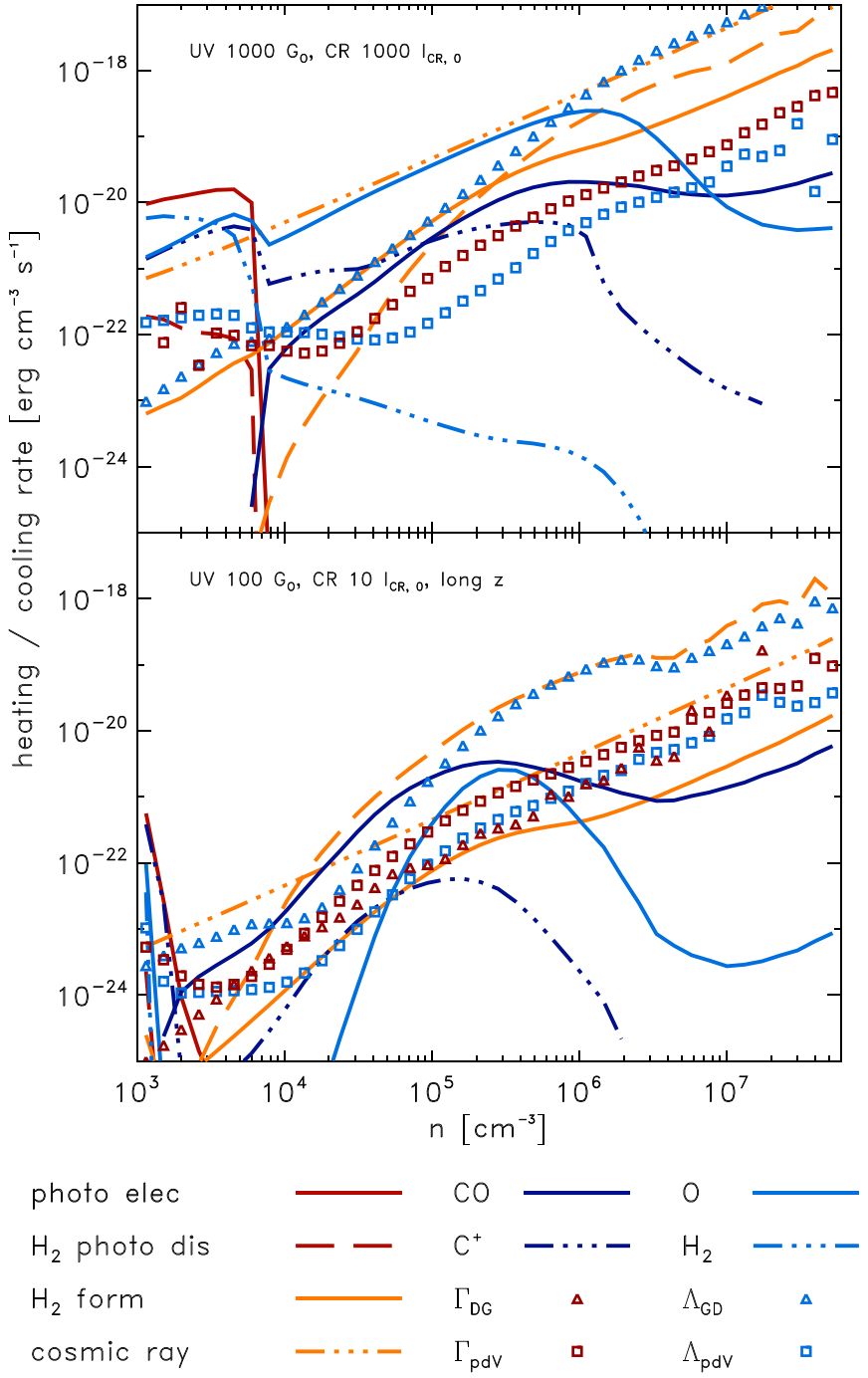

shocks

Figure 4. Processes responsible for heating and cooling the gas in two very different cloud models (clouds 1 and 4 from Table 1). The heating processes are shown in red and orange and the cooling processes are represented in blue. Two processes- $p d V$ work and gas-dust thermal coupling - can produce either heating or cooling depending on the circumstances. Heating and cooling associated with compression and expansion are denoted by $\Gamma_{p d V}$ and $\Lambda_{p d V}$, respectively, while the transfer of energy from the gas to the dust is denoted by $\Lambda_{\mathrm{GD}}$ and that from the dust to the gas by $\Gamma_{\mathrm{DG}}$. The plotted quantities represent the median values at each density.

(A color version of this figure is available in the online journal.)

regime, just as is the case in local molecular clouds. In the denser cloud model, however, CO never dominates; instead, atomic oxygen becomes the main coolant. This difference in behavior is a result of the CRIR in these two clouds. In the higher density cloud, the much higher CRIR creates many $\mathrm{He}^{+}$ ions that react destructively with the $\mathrm{CO}$ molecules:

$$
\mathrm{CO}+\mathrm{He}^{+} \rightarrow \mathrm{C}^{+}+\mathrm{O}+\mathrm{He}
$$

It also keeps the gas warm enough to excite the fine-structure lines of atomic oxygen. In the lower density cloud with the much lower CRIR, both of these effects are less important, and hence atomic oxygen never becomes the dominant coolant. Since we need a large CRIR to explain the observed gas temperatures, the implication is that the cooling of gas in G0.253+0.016 (and probably also in other GC clouds) is dominated over a significant range in densities by emission from atomic oxygen.

At very high densities, dust becomes the most effective source of cooling. However, this does not occur until the gas density is more than an order of magnitude higher than the mean cloud density, and hence we expect that $T_{\text {gas }}=T_{\text {dust }}$ only in the densest gas within G0.253+0.016, with most of the volume of the cloud having $T_{\text {gas }} \neq T_{\text {dust }}$. As already noted, this expectation is supported by the available observational data on the gas and dust temperatures.

The effect of the clouds' environment on the chemical balance is summarized in Table 1. We see that strong ISRFs and CRIRs have little effect on the $\mathrm{H}_{2}$ fraction, and so we would expect the true molecular state of the cloud to be relatively independent of the environment. However, the $\mathrm{CO}$ fraction varies by around an order of magnitude in the models, implying that its ability to trace the molecular state of the gas is a strong function of the environment. Since the clouds initially have all of their carbon in the form of $\mathrm{C}^{+}$, one might argue that we have simply ended our simulations too soon to pick up all of the CO. However, we see that in the clouds with smaller CRIRs, over half of the carbon is in $\mathrm{CO}$, suggesting that there is sufficient time available for it to form in large quantities. As such, the low $\mathrm{CO}$ abundances in the clouds with high CRIR are due to real differences in their chemical evolution.

\section{DISCUSSION}

Our results suggest that the CRIR and ISRF around G0.253+0.016 should be 1000 times the solar neighborhood values, in order to obtain temperatures consistent with the values derived from observations. Such radiation and CR fields could be produced by enhanced star formation activity, higher stellar densities, or some combination of both. Yusuf-Zadeh et al. (2009) measured the star formation rate (SFR) in the GC to be 50-100 times the local SFR. If the CRIR and ISRF are set solely by star formation, our results suggest that the local SFR near G0.253+0.016 is about an order of magnitude higher than the mean SFR of the central molecular zone (Morris \& Serabyn 1996; Yusuf-Zadeh et al. 2009).

Similarly, the CRIR that we require is significantly higher than the values found for local dense clouds. However, there is considerable observational evidence that the ionization rate is higher in the GC. For example, Oka et al. (2005) estimate a value of $2-7 \times 10^{-15} \mathrm{~s}^{-1}$ in diffuse gas along several GC sight lines, while Yusuf-Zadeh et al. (2007) infer a value of $2-50 \times 10^{-14} \mathrm{~s}^{-1}$ within GC clouds, based on observations of the fluorescent $6.4 \mathrm{keV} \mathrm{K} \alpha$ iron line. Our required value of a few times $10^{-14} \mathrm{~s}^{-1}$ is compatible with these values, given the large uncertainties.

Our models also suggest that the neutral oxygen emission coming from G0.253+0.016 should be significantly higher than that seen in typical molecular clouds. This could provide an independent test of the models presented in this Letter.

The authors thank Katharine Johnston and Elizabeth Mills for their enlightening discussions on G0.253+0.016. We acknowledge financial support from the DFG via SFB 811 "The Milky Way System" (sub-projects B1 and B2), and from the BadenWürttemberg-Stiftung by contract research via the programme Internationale Spitzenforschung II (grant P- LS-SPII/18). P.C.C. and S.E.R. are supported by grants CL 463/2-1 and RA 2158/1-1, respectively, which are part of the DFG-SPP 1573. The simulations presented in this Letter were performed on 
the Milkyway supercomputer at the Jülich Forschungszentrum, funded via SFB 811.

\section{REFERENCES}

Ao, Y., Henkel, C., Menten, K. M., et al. 2013, A\&A, 550, A135

Black, J. H. 1994, in ASP Conf. Ser. 58, The First Symposium on the Infrared Cirrus and Diffuse Interstellar Clouds, ed. R. M. Cutri \& W. B. Latter (San Francisco, CA: ASP), 355

Bohlin, R. C., Savage, B. D., \& Drake, J. F. 1978, ApJ, 224, 132

Clark, P. C., Glover, S. C. O., \& Klessen, R. S. 2012, MNRAS, 420, 745

Draine, B. T. 1978, ApJS, 36, 595

Draine, B. T., \& Bertoldi, F. 1996, ApJ, 468, 269

Elmegreen, B. G., Klessen, R. S., \& Wilson, C. D. 2008, ApJ, 681, 365

Glover, S. C. O., \& Clark, P. C. 2012a, MNRAS, 421, 9

Glover, S. C. O., \& Clark, P. C. 2012b, MNRAS, 421, 116

Goldsmith, P. F., \& Langer, W. D. 1978, ApJ, 222, 881

Güesten, R., Walmsley, C. M., \& Pauls, T. 1981, A\&A, 103, 197

Hubber, D. A., Goodwin, S. P., \& Whitworth, A. P. 2006, A\&A, 450, 881

Kruijssen, J. M. D., Longmore, S. N., Elmegreen, B. G., et al. 2013, MNRAS, submitted (arXiv:1303.6286)
Krumholz, M. R., Dekel, A., \& McKee, C. F. 2012, ApJ, 745, 69

Lis, D. C., Menten, K. M., Serabyn, E., \& Zylka, R. 1994, ApJL, 423, L39

Longmore, S. N., Bally, J., Testi, L., et al. 2013, MNRAS, 429, 987

Longmore, S. N., Rathborne, J., Bastian, N., et al. 2012, ApJ, 746, 117

Mathis, J. S., Mezger, P. G., \& Panagia, N. 1983, A\&A, 128, 212

Morris, M., \& Serabyn, E. 1996, ARA\&A, 34, 645

Nelson, R. P., \& Langer, W. D. 1999, ApJ, 524, 923

Oka, T., Geballe, T. R., Goto, M., Usuda, T., \& McCall, B. J. 2005, ApJ, 632,882

Ossenkopf, V., \& Henning, Th. 1994, A\&A, 291, 943

Padovani, M., Galli, D., \& Glassgold, A. E. 2009, A\&A, 501, 619

Papadopoulos, P. P. 2010, ApJ, 720, 226

Rodríguez-Fernández, N. J., Martín-Pintado, J., Fuente, A., \& Wilson, T. L. 2004, A\&A, 427, 217

Schleicher, D. R. G., Spaans, M., \& Klessen, R. S. 2010, A\&A, 513, A7

Springel, V. 2005, MNRAS, 364, 1105

van der Tak, F. F. S., \& van Dishoeck, E. F. 2000, A\&A, 358, L79

Walmsley, C. M., \& Ungerechts, H. 1983, A\&A, 122, 164

Yusuf-Zadeh, F., Hewitt, J. W., Arendt, R. G., et al. 2009, ApJ, 702, 178

Yusuf-Zadeh, F., Wardle, M., \& Roy, S. 2007, ApJL, 665, L123 